\title{
Maintain and Regain Well Clear: Maneuver Guidance Designs for Pilots Performing the Detect-and-Avoid Task
}

\author{
Kevin J. Monk ${ }^{1, *}$ and Zachary Roberts ${ }^{2}$ \\ ${ }^{1}$ NASA Ames Research Center \\ 94035 Moffett Field, USA \\ kevin.j.monk@nasa.gov \\ ${ }^{2}$ San Jose University Research Foundation \\ 94035 Moffett Field, USA \\ zachary.s.roberts@nasa.gov
}

\begin{abstract}
In order to support the future expansion and integration of Unmanned Aircraft Systems (UAS), ongoing research efforts have sought to produce findings that inform the minimum display information elements required for acceptable UAS pilot response times and traffic avoidance. Previous simulations have revealed performance benefits associated with DAA displays containing predictive information and suggestive maneuver guidance tools in the form of banding. The present study investigated the impact of various maneuver guidance display configurations on detect-and-avoid (DAA) task performance in a simulated airspace environment. UAS pilots' ability to maintain DAA well clear was compared between displays with either the presence or absence of green DAA bands, which indicated conflict-free flight regions. Additional display comparisons assessed pilots' ability to regain DAA well clear with two different guidance presentations designed to aid in DAA well clear recovery during critical encounters. Performance implications and display considerations for future UAS DAA systems are discussed.
\end{abstract}

Keywords: Human-systems Integration · Human Factors · UAS · User Interface Design $\cdot$ Detect and Avoid $\cdot$ Safety $\cdot$ Aviation $\cdot$ Aeronautics $\cdot$ Displays

\section{$1 \quad$ Introduction}

Current day applications of Unmanned Aircraft Systems (UAS) primarily involve military operations in restricted airspace. However, civil and public-use UAS are expected to fly alongside manned commercial aircraft unrestricted across other airspace classes within the National Airspace System (NAS) in the coming years. Subject matter experts 
from academia, industry, and government are currently developing minimum operational performance standards (MOPS) in order to maintain safety of flight with the integration of UAS in the NAS [1][2]. Currently, federal regulations require manned pilots to "see and avoid" other aircraft to remain "well clear" [3]. Since UAS pilots are positioned at a ground control station (GCS) without the ability to visually detect potential threats from inside the cockpit, they will require a "detect and avoid" (DAA) system that provides the information necessary to identify a threat and make an appropriate maneuver with the command and control interface [4]. The minimum amount of information on the DAA display needed to detect conflicts, determine a resolution, and avoid losses of well clear safely has been the focus of ongoing research within NASA's UAS integration in the NAS (UAS-NAS) project.

Previous research has explored the minimum DAA display requirements necessary to perform UAS pilot tasks. Predictive displays that include avoidance zones, colorcoded alerting, intruders' relative closest-point-of-approach (CPA), and directional icons with conflict alerting have been shown to reduce losses of DAA well clear (DWC) and minimize the severity of collision hazards when they do occur [5][6]. A survey assessing pilots' visual information preference identified intruder state information, visual alerts, and DAA maneuver recommendations as important information elements [7].

Human-in-the-loop simulations have revealed performance benefits with displays containing advanced conflict resolution tools integrated into the vehicle control interface on the ground control station (GCS) [8][9][10]. Specifically, suggestive maneuver guidance in the form of banding that provided continuous indications of the predicted threats (i.e., losses of DWC) at nearby headings and altitudes have yielded the most desirable benefits compared to an informative display [11][12]. While DAA banding guidance that indicated the predicted threat severity level was accepted as a requirement in the Phase 1 MOPS for the DAA system, it was not specified whether it is necessary to incorporate green, conflict-free bands that highlight flight regions absent of any predicted loss of DWC threats. Furthermore, the suggestive maneuver guidance in previous evaluations informed maneuvers for maintaining well clear with sufficient time, but did not always provide guidance to aid in regaining well clear in the more severe cases where a near midair collision (NMAC) was imminent. Recovery guidance presented to pilots was not directly assessed for its effects, and there were too few losses of DWC with the banding displays to make post-hoc statistical comparisons. Therefore, the present study utilized a test setup that allowed for a more complete evaluation of the conflict-free maneuver guidance bands and various well clear recovery guidance design concepts with respect to pilots' ability to maintain and regain well clear.

\section{$2 \quad$ Method}

\subsection{Participants}

Ten pilots were recruited for participation in the current experiment. Six of ten were active duty UAS pilots $\left(M_{\text {age }}=36\right.$ years old), with averages of 1600 hours of manned flight experience and 1400 hours of unmanned flight experience. The other four were commercial pilots ( $M_{\text {age }}=30$ years old) with an average of 9000 hours of manned flight experience in civil airspace. 


\subsection{Simulation Environment}

Ground Control Station. The GCS used for this study was the Vigilant Spirit Control Station (VSCS), which was developed by the Air Force Research Laboratory (AFRL) [13]. For the purposes of the current study, VSCS provided a Tactical Situation Display (TSD) that displayed ownship, mission route, DAA maneuver guidance, and traffic information over a moving map. The TSD supported an autopilot control interface that allowed pilots to change their altitude and heading without manipulating the pre-filed flight plan route. Heading holds could be executed via the graphical compass rose interface or keypad inputs to a steering command window. The compass rose interface allowed pilots to click-and-drag an arrow shaped heading bug to the desired heading rather than manually input numbers. Altitude values could be changed either by manually typing in desired values or by using two small arrows ("spinners") that would increase/decrease altitudes by 500 feet (ft.) per mouse click. Pilots uploaded commands to the aircraft by clicking the "Send" button located within the steering window. On a separate monitor only visible by the researcher, another component of the Vigilant Spirit software ('Vigilant Spirit Simulation') allowed researchers to manually launch the pre-scripted encounters toward the ownship.

DAA System. The multi-level alerting structure was constructed through the Java Architecture for DAA Modeling and Extensibility (JADEM v.5.4.1) [14]. Intruders equipped with transponders (i.e., cooperative) were displayed on the TSD at sensor ranges of 15 nautical miles (nmi) laterally and $\pm 5000 \mathrm{ft}$. vertically, while intruders with RADAR-only equipage (i.e., non-cooperative) were detected at a lateral range of $8 \mathrm{nmi}$ with the same vertical range. Color-coded symbology was applied to all aircraft within sensor range to provide pilots with indications of individual threat severity, based on whether they were currently predicted to penetrate the spatial and temporal thresholds pre-defined for the current study (see Table 1). Direct auditory alerts were presented as the threat severity levels of the intruders increased. The intruders' relative altitude and vertical trend were also constantly visible once they were within sensor range. Other intruder elements that appeared within the data tag at the onset of a conflict alert included call sign (cooperative intruders only), ground speed, absolute altitude, and vertical velocity.

The DAA maneuver guidance ('Omni Bands'), also generated by the JADEM DAA system, provided pilots with a form of conflict resolution using dashed lines ('banding') that predicted whether particular heading or altitude values were predicted to cause loss of DWC threats if flown at that time. The horizontal bands probed relative headings within $270^{\circ}$ around ownship and appeared on the inner range ring of the moving map. The vertical bands probed altitudes within $\pm 2,000 \mathrm{ft}$. of ownship on the altitude tape to the right of the TSD. The maneuver guidance bands were constantly updating to reflect the most up-to-date flight state information, as JADEM did not account for ownship or intruder intent. The heading and altitude bands were color-coded in correspondence with the predicted threat level from the alerting structure. Headings and altitudes with yellow bands were predicted to lead to a loss of DWC (as defined in Table 1) with an intruder aircraft within 25 to 55 seconds. Red banding indicated that a particular heading or altitude would lead to a loss of DWC within 25 seconds or less. Thus, regions with yellow or red banding were to be avoided, as maneuvers toward these areas would 
trigger at least one Corrective DAA or DAA Warning alert, respectively. Safe flight regions that would remain well clear with intruders were indicated by either the presence of green banding or the absence of banding, depending on the condition (see Experimental Design).

Table 1. Multi-Level Alerting Scheme

\begin{tabular}{|c|c|c|c|c|}
\hline Alert Level & $\begin{array}{c}\text { Separation } \\
\text { Criteria }\end{array}$ & $\begin{array}{c}\text { Time to } \\
\text { Loss of } \\
\text { DWC }\end{array}$ & Icon & $\begin{array}{c}\text { Aural Alert } \\
\text { Verbiage }\end{array}$ \\
\hline $\begin{array}{c}\text { DAA Warning } \\
\text { Alert }\end{array}$ & $\begin{array}{c}\mathrm{HMD}=0.75 \mathrm{nmi} \\
\mathrm{ZTHR}=450 \mathrm{ft} . \\
\text { modTau }=35 \mathrm{sec}\end{array}$ & $25 \mathrm{sec}$ & $\begin{array}{c}\text { "Traffic, Maneuver } \\
\text { Now, } \\
\text { Traffic, Maneuver } \\
\text { Now" }\end{array}$ \\
\hline $\begin{array}{c}\text { Corrective } \\
\text { DAA Alert }\end{array}$ & $\begin{array}{c}\mathrm{HMD}=0.75 \mathrm{nmi} \\
\mathrm{ZTHR}=450 \mathrm{ft} . \\
\text { modTau }=35 \mathrm{sec}\end{array}$ & $55 \mathrm{sec}$ & $\mathbf{A}$ & "Traffic, Avoid" \\
\hline $\begin{array}{c}\text { Preventive } \\
\text { DAA Alert }\end{array}$ & $\begin{array}{c}\mathrm{HMD}=0.75-1.0 \mathrm{nmi} \\
\mathrm{ZTHR}=450-700 \mathrm{ft} . \\
\text { modTau }=35 \mathrm{sec}\end{array}$ & N/A & $\mathbf{W}$ & "Traffic, Monitor" \\
\hline None (Target) & $\begin{array}{c}\text { Within surveillance } \\
\text { field of regard }\end{array}$ & N/A & $\mathbf{A}$ & N/A \\
\hline
\end{tabular}

Once resolution options for remaining well clear were no longer achievable by ownship, the bands would fully saturate to red and well clear recovery guidance was presented on the TSD. Though likely that the well clear boundary had already been penetrated in these worst-case scenarios, it was considered necessary to provide pilots with some form of maneuver guidance as a last resort to help minimize the severity of the separation loss and regain DWC. In order to achieve this, the well clear recovery guidance calculated the direction that would lead to the maximum separation at the CPA. The underlying computations used to suggest conflict resolutions were based on the Generic Resolution Advisor and Conflict Evaluator (GRACE) algorithm [15], which evaluates multiple intruders for threats based on the aforementioned separation standards. The GRACE maneuver selection logic generated conflict-free solutions with considerations made to current intruder flight states and, in well clear recovery cases, presented either the lateral or vertical maneuver suggestion with the lowest 'NMAC cost'.

The recovery guidance was displayed to pilots both textually and graphically on the TSD. The graphical representation of the maneuver recommendation varied among trials (see Experimental Design). The textual guidance for both recovery displays was shown at the top of the TSD inside of a green border, labeled with commands of either 'Turn Right', 'Turn Left', 'Climb', or 'Descend'. The recovery guidance text would switch to 'Maintain' to inform pilots that they may remain at their current heading and altitude for the time being once they reached the flight state necessary to maximize their separation with the surrounding intruder(s). Once ownship regained well clear with the 
intruder(s) and the bands were no longer saturated red, the recovery guidance as a whole was no longer displayed.

\subsection{Experimental Design}

The current experiment utilized a one-way and repeated measures design to examine the impact of green DAA bands (With vs. Without) and band saturation display options (Limited Suggestive vs. Directional) on pilots' DAA task performance.

Green DAA Bands. In the previous simulation that introduced Omni Bands [11], green banding was used to denote safe flight regions that would not result in a loss of DWC if flown at that time. Headings and altitudes that were not probed (i.e., the $90^{\circ}$ behind ownship, $>3,000 \mathrm{ft}$ relative altitude) did not have any banding. During the open-ended portion of the debriefs, pilots voiced varied opinions on the usefulness of the green bands for conflict avoidance, with some stating that they added display clutter in conditions using Omni Bands (albeit quantitative analysis of questionnaire responses with regard to display clutter did not reveal statistical significance). The present study sought to examine whether the presence (or absence) of green DAA banding had any impact on pilot performance and response times; thus, it was added as a between-subjects manipulation. Half of the pilots saw green DAA bands which differentiated safe regions from those that were un-probed or would create conflict (at the Corrective DAA or DAA Warning levels), while the others did not have green DAA bands displayed to them (Fig. 1). The pilots that did not have use of the green bands saw a blank, defaulted (grey) presentation of their inner range ring and altitude tape (similar to when unprobed) until there was a potential conflict within sensor range that triggered yellow and/or red bands on the display. Pilots without green DAA bands were instructed to avoid conflicts by flying into regions with no banding present.
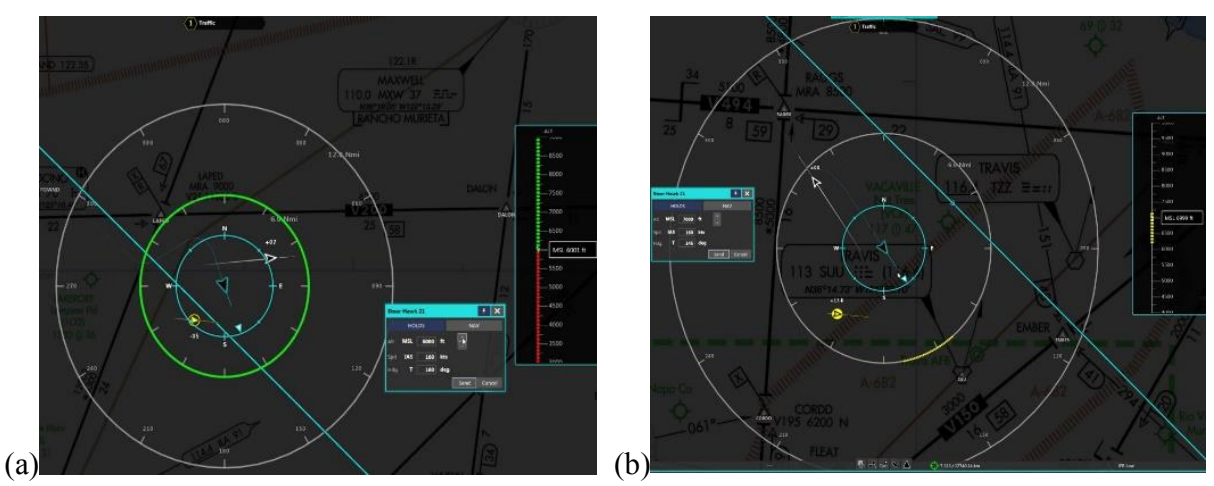

Fig. 1. TSD with (a) and without (b) green bands displayed.

Well Clear Recovery Guidance. The Well Clear Recovery guidance display(s) appeared when a loss of DWC could no longer be avoided. There were two graphical representations of guidance presented to pilots for regaining well clear: Limited Suggestive and Directional. The Limited Suggestive recovery guidance displayed the range of optimal headings or altitudes to fly in order to maximize separation (Fig. 2). Low 
and high bounds of a recommended altitude or heading range were provided to achieve a timely regain of well clear. A green 'wedge' encompassing the suggestion range appeared next to ownship and extended out to the range rings when the algorithm recommended a turn; pilots were to comply by flying to headings within the suggestive wedge. If the recovery algorithm recommended a vertical maneuver for collision avoidance, pilots were to aim for altitudes within the green wedge that appeared on the altitude tape.

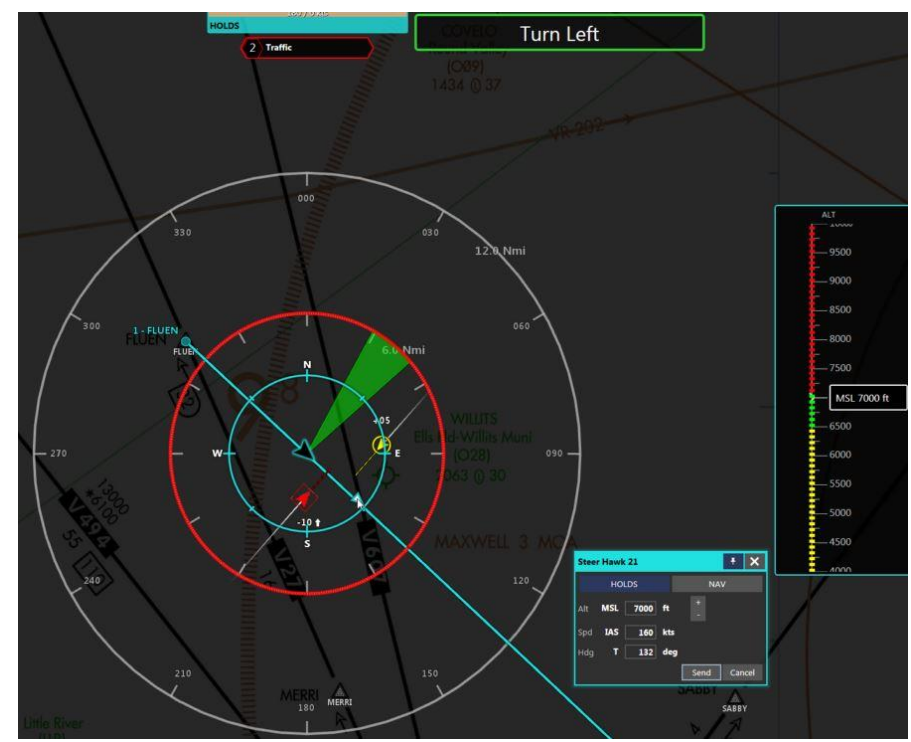

Fig. 2. Limited Suggestive Well Clear Recovery Guidance.

The Directional recovery guidance simply indicated the suggested maneuver type by displaying an arrow in the recommended direction of the maneuver (Fig. 3). A green arrow appeared pointing to either the left or right of ownship when the recovery guidance was recommending a turn. For vertical maneuver recommendations, an up or down arrow appeared to the left of the altitude tape to suggest a climb or descent. Directional recovery guidance did not specify a specific range of headings or altitudes to choose from, instead allowing the pilot to determine the size of the maneuver in the recommended direction and sense. 


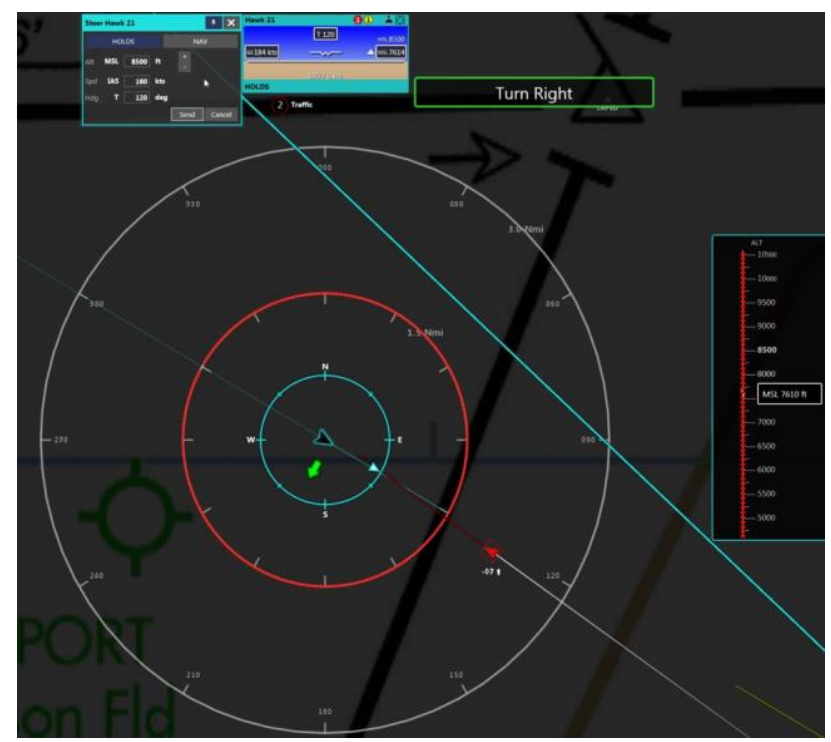

Fig. 3. Directional Well Clear Recovery Guidance.

\subsection{Procedure}

Training. Pilots began the day by filling out demographics and informed consent forms. They were then given a short briefing on the experiment before being trained on the basic functionalities within VSCS. Once pilots demonstrated proficiency with the vehicle control inputs required to maneuver the simulated aircraft, they were trained on the various components of the DAA system (above). Pilots assigned to the display configuration with no green bands for remaining well clear were trained with slides that excluded any mention of green bands. Each experimental scenario was preceded by a training run that allowed pilots to practice interaction with the upcoming well clear recovery display configuration. The banding options for remaining well clear varied between subjects, while the recovery display options varied between trials (within subjects).

DAA Pilot Task. Pilots completed four 40-minute scenarios --- two with each well clear recovery guidance display. The order of presentation was counterbalanced between participants. Pilots were instructed to navigate a simulated MQ-9 Reaper along a route line while avoiding well clear violations and NMACs with nearby intruders. The scenarios consisted of 20 encounters lasting approximately two minutes each. Sixteen of the 20 encounters were scripted to lose well clear absent any pilot action, with eight of them involving conflicts that blundered into ownship and forced an immediate well clear violation. Since there were hardly any losses of DWC observed for displays utilizing green DAA banding in the previous simulation [11], it was necessary to introduce severe encounters that allowed for the evaluation of pilots' ability to regain well clear with each recovery band display option. Pilots were instructed not to begin editing 
their trajectory until the onset of a DAA alert required them to do so. Once pilots complied with guidance and successfully avoided an aircraft, they were to return to course and fly along the route line until their next encounter was triggered.

\subsection{Measures}

Initial Response Time (Initial RT). Initial Response Time refers to the amount of time it took for pilots to initiate a navigational edit into the GCS after the onset of a Corrective DAA or DAA Warning alert.

Total Edit Time. Total Edit Time refers to the amount of time it took for pilots to complete their final upload into the navigation interface after starting their initial edit.

Total Response Time (Total RT). Total Response Time refers to the full amount of time it took for pilots to upload their final resolution after the onset of a Corrective DAA or DAA Warning alert.

Loss of DWC (LoDWC) Severity. As of the present study, the overall severity of each well clear violation was identified by the DAA Well Clear Penetration Integral (DWCPI) metric [16], which combined the amount of time spent within the well clear threshold and the minimum geometric separation at CPA into a single measure. The greater the DWCPI magnitude for a given encounter, the more severe the loss of DWC event was considered.

\section{$3 \quad$ Results}

\subsection{Green DAA Bands}

The response time metrics were analyzed across the two banding displays using a oneway Analysis of Variance (ANOVA), with an alpha level of .05. The analysis included the encounter cases that required pilots to remain DWC (i.e., when positive maneuver guidance bands were available). The LoDWC severity results for this variable were not tested for statistical significance, as there was only one loss of DWC occurrence among all nominal encounters with each banding display.

Initial RT. There was a significant main effect of green bands on initial response times, $F(1,281)=13.10, p<.05$. Initial RTs were, on average, 1 second $(s)$ quicker with the No Green Bands display $(M=5.00 s, S E=0.32 s)$ compared to the Green Bands display $(M=6.00 s, S E=0.25 s)$.

Total Edit. There was only a marginal effect of green bands on total edit times, $F(1$, $283)=3.74, p=.054$. Pilot completed their edits, on average, $0.86 s$ quicker with the Green Bands display $(M=3.87 s, S E=0.26 s)$ compared to the No Green Bands display $(M=4.73 s, S E=0.37 s)$. 
Total RT. There was no significant effect of green bands found on total response time, $p>.05$.

\subsection{Well Clear Recovery Type}

The response time and separation metrics were analyzed across the two well clear recovery displays using a repeated measures ANOVA, with an alpha level of .05. Statistical comparisons were made across encounters that presented well clear recovery guidance to regain well clear, including the critical cases that blundered into ownship.

Initial RT. There was no significant difference in initial response times found between the Limited Suggestive $(M=3.98 s, S E=0.59 s)$ and Directional $(M=3.61 s, S E=0.36 s)$ display configurations, $p>.05$.

Total Edit. There was no significant difference in total edit times found between the Limited Suggestive $(M=5.20 s, S E=1.32 s)$ and Directional $(M=5.71 s, S E=1.44 s)$ display configurations, $p>.05$.

Total RT. There was no significant difference in total response times found between the Limited Suggestive $(M=9.17 s, S E=1.25 s)$ and Directional $(M=9.31 s, S E=1.32 s)$ display configurations, $p>.05$.

LoDWC Severity. While loss of DWC events were, on average, slightly less severe with the Limited Suggestive display configuration $(M=0.86, S E=0.19)$ compared to the Directional display $(M=1.34, S E=0.32)$, the difference in DWCPI magnitude was nonsignificant, $p>.05$.

\section{Discussion}

\subsection{Conflict-free Bands for Maintaining DAA Well Clear}

The results suggest that DAA guidance in the form of banding is effective at aiding the pilot responsibility of remaining well clear, regardless of whether green bands are implemented to highlight the well clear regions. Initial RT was the only response time metric that yielded a (significant) difference of over one second between conditions. Pilots utilizing the green bands started their initial edits following a DAA Corrective alert $1.5 \mathrm{~s}$ slower on average. There may be slightly less of a processing delay when simply monitoring the onset of conflict bands versus detecting continuous changes in the color of bands constantly visible on the display. Nonetheless, conflicts were successfully avoided at a nearly equal rate across banding displays overall. There was only 1 LoDWC ( $<1 \%$ of total encounters) with each banding display, and the LoDWC proportion was comparable to previous analyses observing non-blunders at nominal encounter ranges sufficient to remain well clear [16]. The Phase 1 DAA MOPS require a distinction between the yellow corrective and red warning guidance bands, while the implementation of green (or any color) conflict-free maneuver guidance bands are considered optional [17]. 


\subsection{Recovery Guidance for Regaining DAA Well Clear}

Well clear recovery display type failed to significantly impact any of the response time or separation variables in the present study. Response times were nearly identical between recovery displays, as there was a difference of a half-second or less on every response time metric. It should be noted that no large response time differences between recovery types were expected, as well clear recovery did not appear until the pilot could no longer maintain DAA well clear. Once the bands were fully saturated red and the threat severity reached the critical Warning level, an immediate maneuver in compliance with the well clear recovery bands was the expected pilot action. While pilots were trained to comply with the guidance, it was left to their discretion whether it was deemed appropriate. Minimal decision-making was required when pilots made immediate maneuvers in compliance with the guidance as expected. Pilots complied with the well clear recovery guidance to regain well clear in 359 of the 365 (98\%) LoDWC occurrences. Five of the 6 non-compliance cases involved vertical resolution uploads being made instead of the recommended turn (possibly in anticipation of a subsequent vertical resolution advisory), and one was due to the pilot preferring a turn in the opposite direction. Compliance rates were identical between displays. Subjective ratings gathered from post-simulation questionnaires were also nearly equal between the Limited Suggestive and Directional displays (preferred by $60 \%$ and $40 \%$ pilots, respectively).

Loss of DWC events were slightly less severe when using the Limited Suggestive guidance, which presented a specific solution range to pilots at the onset of recovery bands. While the precise recommendations slightly decreased the time spent within the well clear threshold, differences in LoDWC severity were not significant. The Limited Suggestive and Directional well clear recovery guidance displays available in the present study reduced LoDWC severity by $78 \%$ and $64 \%$, respectively, compared to the previous analysis of the DAA system without recovery guidance [16]. The recovery displays appear to be equally effective at aiding the pilot task of regaining DAA well clear against intruders at critical ranges, and both were referenced as viable guidance options for maximizing horizontal and/or vertical miss distance during a loss of DWC event in Phase 1 of the DAA MOPS [17]. In conclusion, multiple design concepts are acceptable for maintaining and regaining DWC when the guidance corresponds with the alerting logic.

\section{$5 \quad$ References}

1. RTCA: Terms of reference: RTCA special committee 228 minimum performance standards for unmanned aircraft systems. RTCA Inc., Washington, DC (2013)

2. Federal Aviation Administration (FAA): Integration of civil UAS in the NAS roadmap, first edition. FAA, Washington, DC (2013)

3. Code of Federal Regulations: 14 CFR, Part 91, Sec. 91.113 (2004)

4. Santiago, C. \& Mueller, E.R.: Pilot evaluation of a UAS detect-and-avoid system's effectiveness in remaining well clear. 11th USA/Europe Air Traffic Management Research and Development Seminar, Lisbon, Portugal (2015) 
5. Bell, S., Drury, J., Estes, S., \& Reynolds, C.: GDTI: A ground station display of traffic information for use in sense and avoid operations. Digital Avionics Systems Conference (DASC), 2012 IEEE/AIAA 31st, Williamsburg, VA (2012)

6. Friedman-Berg, F., Rein, J. \& Racine, R.: Minimum visual information requirements for detect and avoid in unmanned aircraft systems. Proceedings of the Human Factors and Ergonomics Society 58th Annual Meeting, Chicago, IL (2014)

7. Draper, D.H., Pack, J.S., Darrah, S.J., \& Moulton, S.N.: Human-machine interface development for common airborne sense and avoid program. Proceedings of the Human Factors and Ergonomics Society 58th Annual Meeting, Chicago, IL (2014)

8. Fern, L., Rorie, R.C., Pack, J., Shively, R.J., \& Draper, M. “An evaluation of DAA displays for unmanned aircraft systems: The effect of information level and display location on pilot performance." Proceedings of 15th AIAA Aviation Technology, Integration, and Operations Conference, Dallas, TX (2015)

9. Rorie, R. C. \& Lisa Fern: "The impact of integrated maneuver guidance information on UAS pilots performing the Detect and Avoid task." In Proceedings of the 59th Human Factors and Ergonomics Society Annual Meeting (2015)

10. Monk, K.J., Fern, L., Rorie, R.C., \& Shively, R.J.: Effects of display location and information level on UAS pilot assessments of a detect and avoid system. Proceedings of the Human Factors and Ergonomics Society 59th Annual Meeting, Los Angeles, CA (2015)

11. Rorie, R. C., Fern, L., \& Shively, J.: The impact of suggestive maneuver guidance on UAS pilot performing the detect and avoid function. AIAA Infotech@ Aerospace, San Diego, CA (2016)

12. Monk, K.J. \& Roberts, Z.: UAS Pilot Evaluations of Suggestive Guidance on Detect-andAvoid Displays. Proceedings of the Human Factors and Ergonomics Society 60th Annual Meeting, Washington, DC (2016)

13. Feitshans, G. L., Rowe, A. J., Davis, J. E., Holland, M., \& Berger, L.: Vigilant spirit control station (VSCS) - 'The face of COUNTER'. Proceedings of AIAA Guidance, Navigation and Control Conf. Exhibition, Honolulu, HI (2008)

14. Santiago, C., Abramson, M., Refai, M., Mueller, E., Johnson, M., \& Snow, J.: Java architecture for detect and avoid (DAA) modeling and extensibility (JADEM) (2015)

15. Abramson, M., Mohamad Refai, and Confesor Santiago: "A Generic Resolution Advisor and Conflict Evaluator (GRACE) in Applications to Detect-And-Avoid (DAA) Systems of Unmanned Aircraft". Proceedings of 17th AIAA Aviation Technology, Integration, and Operations Conference (2017)

16. Mueller, E., Santiago, C., and Watza, S.: "Piloted "Well Clear" Performance Evaluation of Detect-and-Avoid Systems with Suggestive Guidance," NASA/TM-2016-219396 (2016)

17. RTCA. "Minimum operational performance standards (MOPS) for unmanned aircraft systems (UAS) detect and avoid (DAA) systems" RTCA Inc., Washington, DC (2017) 